\title{
Creación de una Estructura Colaborativa entre el Alumnado, Docentes e Investigadores: Impacto en la Mejora Docente
}

\section{Creation of a Collaborative Structure between Students, Teachers and Researchers: Impact on Teaching Improvement}

\author{
Cecilia Simón * \\ Gerardo Echeita \\ Marta Sandoval \\ $\mathrm{M}^{\mathrm{a}}$ José de Dios
}

Universidad Autónoma de Madrid, España

\begin{abstract}
El trabajo que se presenta tiene como objetivo mostrar la eficacia de la creación de una estructura de diálogo entre estudiantes y profesorado con la finalidad de mejorar las prácticas docentes en el sentido de articular con equidad la presencia, el aprendizaje y la participación de todo el alumnado sin dejar a nadie atrás. Se trata de un proyecto de investigación-acción en el que se ha contado con la participación de seis centros de educación primaria. En cada centro se ha implicado alumnado y docentes de diferentes cursos y materias, contándose con la participación voluntaria de 18 docentes y 52 estudiantes investigadores, formando parte del estudio un total de 201 alumnos y alumnas. Se ha partido de un modelo de "investigación inclusiva" en el que se han incorporado estrategias de participación de los estudiantes como investigadores y colaboración entre docentes. La información sobre el impacto de las lecciones de investigación se ha recogido a través de entrevistas, grupos de discusión, observación en clase y cuestionarios. Los resultados muestran la repercusión de estas estrategias en la mejora docente, en las concepciones y actitudes de los docentes respecto a la participación de los estudiantes y en el propio alumnado que ha participado como investigador.
\end{abstract}

Descriptores: Prácticas inclusivas; Desarrollo docente; Estudiantes investigadores; Colaboración entre docentes; Voces de estudiantes.

The aim of the work presented here is to show the effectiveness of creating a structure for dialogue between students and teachers with the aim of improving teaching practices in order to ensure the presence, learning and participation of all learners without leaving anyone behind. This is an action-research project in which six primary schools have participated. Each school involved learners and teachers from different academic courses and subjects, with the voluntary participation of 18 teachers and 52 student researchers, with a total of 201 learners taking part in the study. The starting point was a model of "inclusive research" in which strategies for student participation as researchers and collaboration among teachers were incorporated. Information on the impact of the research lessons was collected through interviews, focus groups, classroom observation and questionnaires. The results show the effect of these strategies on teacher professional learning, on teachers' beliefs and attitudes towards student participation and on the students, themselves who have participated as researchers.

Keywords: Inclusive practices; Teacher professional learning; Student researchers; Teacher collaboration; Students' voices.

*Contacto: cecilia.simon@uam.es

ISSN: 0718-7378

www.rinace.net/rlei/
Recibido: 12/07/2021

$1^{\text {a }}$ Evaluación: 10/09/2021

Aceptado: $\quad 03 / 10 / 2021$ 


\section{Introducción}

El derecho a una educación inclusiva requiere de cambios sistémicos que afectan no solo a las escuelas, sino también a ámbitos situados más allá de las mismas, referidos a elementos propiamente estructurales del sistema educativo y de su concreción nacional o local, así como otros que tienen que ver, por ejemplo, con variables de orden socioeconómico, cultural, urbanístico, laboral o relativas a la salud que afectan a la infancia y a las familias (Ainscow et al., 2013). Sin obviar esto, a pesar de los muchos propósitos internacionales (UNESCO, 2015) y de los avances innegables realizados (UNESCO 2020a), las dificultades para concretar este derecho en las aulas, de forma que la actuación docente llegue a todo el alumnado, son evidentes (UNESCO, 2020b). Sabemos las claves que, en términos de acciones específicas, pueden ayudar a los sistemas educativos a avanzar en esta dirección. Una de las que señala la UNESCO (2020a, p. 28) relacionada con el objetivo de este trabajo es "Velar por que se preste apoyo a los docentes en su acción de promoción de la inclusión y la equidad". En esta acción se reconoce el papel fundamental de la formación previa, así como del desarrollo profesional permanente. Aspecto, este último, objeto de atención en diferentes trabajos, como el realizado por la Agencia Europea para las Necesidades Especiales y la Inclusión Educativa en 2012 con el objetivo de delimitar el perfil de un profesorado para la inclusión.

En este sentido, asumir la responsabilidad de la formación continua se considera como un principio básico que debe fundamentar el desempeño profesional de los docentes. De la misma forma, sabemos que las escuelas preocupadas por la inclusión comparten un elemento en común como es el de cuidar este desarrollo profesional (Porter y Towell, 2019) y buscar los recursos, tanto dentro como fuera de la escuela, para ello. Precisamente, en este trabajo se pretende mostrar una estrategia de mejora de la práctica docente utilizando como recurso las fortalezas existentes en la propia escuela.

El estudio que se presenta se enmarca en un proyecto financiado por la Unión Europea, "Reaching the hard to reach: Respuestas inclusivas a la diversidad a través del diálogo alumnos-profesores" en el que participan universidades y centros educativos públicos de Educación Primaria de cinco países (Austria, Dinamarca, España, Reino Unido y Portugal). El objetivo del proyecto ha sido desarrollar estrategias educativas para el aprendizaje y la participación de todo el alumnado en el aula, con una preocupación particular por aquellos a los que el profesorado considera más "difíciles de llegar", por distintas razones y circunstancias (Messiou y Ainscow, 2020).

Este proyecto parte de un modelo de "investigación inclusiva colaborativa" generado en un trabajo anterior llevado a cabo en centros de educación secundaria de tres países (España, Reino Unido y Portugal), también financiado por la Unión Europea (Messiou et al., 2016). El proyecto actual, además, busca comprobar la eficacia de este modelo colaborativo en las escuelas analizando su impacto en la participación de los estudiantes, en el pensamiento y en las prácticas de los docentes. Al mismo tiempo, tiene por objetivo la capacitación del alumnado como investigadores para ayudar a desarrollar prácticas inclusivas en las escuelas.

La finalidad es que cada escuela identifique la manera de lograr que "todos" sus alumnos y alumnas aprendan al máximo y participen de forma activa en un contexto de relaciones positivas con sus iguales, sin perder de vista en ese "todos" a los estudiantes que tradicionalmente se ha encontrado en situaciones de especial vulnerabilidad a la exclusión. 
El núcleo de la propuesta es establecer vías de diálogo recurrentes entre el alumnado y el profesorado en relación con cómo mejorar las prácticas docentes.

Los ejes conceptuales del modelo que ha guiado el trabajo llevado a cabo se explican con más detenimiento en el artículo presentado por Messiou y Ainscow (2021) en este mismo monográfico. Sin embargo, quisiéramos detenernos en dos elementos básicos del mismo.

En primer lugar, contar con "la voz del alumnado" y promover su participación como investigadores en el proceso. Las escuelas eficaces (McLeskey et al., 2014) se caracterizan por promover actitudes y prácticas de franca participación e implicación de toda su comunidad educativa. En este sentido, los estudiantes pueden ser un recurso de gran valor para la mejora de los centros escolares en general (Fielding, 2018; Susinos et al., 2018) y de la práctica docente en particular (Messiou, 2019; Messiou et al., 2016; Simón et al., 2018). Ello ocurre porque las voces del alumnado proporcionan evidencias que pueden ser una excelente base para una reflexión compartida. Decimos pueden, porque es bien sabido que no todas las formas de participación de los estudiantes tienen el mismo potencial a la hora de generar mejoras escolares (Fielding, 2018; Morris, 2019).

El segundo elemento básico de la propuesta tiene que ver con la importancia de la colaboración entre docentes como estrategia de apoyo para la mejora. De acuerdo con Martín y Cervi (2006), para poder cambiar es necesario indagar sobre las propias concepciones mediante la reflexión, individual y colectiva, y contrastar con los otros el conocimiento profesional para poder transformarlo. Para ello, es importante crear oportunidades en las que sea posible la reflexión en y sobre la práctica. Cuando este proceso de reflexión acción, que implica hacerse preguntas y tomar decisiones, se lleva a cabo junto con otros compañeros o compañeras desde una meta común, el impacto, en cuanto al cambio en las prácticas del equipo docente, no solo puede ser mayor, sino que tiene más posibilidades de ser sostenible.

Lo anterior implica reconocer, en primera instancia, el conocimiento que ya existe en el centro, en cada aula, en cada docente (Harris et al., 2018). El problema es que en muchas ocasiones este conocimiento y experiencia no se reconoce, no se valora o no se generan oportunidades para que los docentes los compartan. En este proyecto esto se ha revertido mediante lo que se han denominado "lecciones de investigación", y que se fundamenta en la estrategia de desarrollo profesional conocida como "lecciones de estudio" (Soto et al., 2019). Contamos con evidencias que muestran su eficacia en el desarrollo profesional docente en general y para la mejora de las prácticas inclusivas en particular (Norwich et al., 2021). Estas "lecciones", son las sesiones que los docentes programan habitualmente para trabajar en torno a determinados contenidos, pero que se convierten en "lecciones de investigación”. Para todas sus fases (diseño, aplicación y evaluación) se genera un diálogo entre todos los involucrados que, en esta ocasión, han sido tríos de docentes, que pueden serlo del mismo ciclo o de cualquier curso de la etapa; los investigadores de las universidades (que se involucran como observadores y "amigos críticos") y el alumnado, seleccionado al efecto con la función de ser investigadores y analistas de tales lecciones. En el artículo presentado por Messiou y Ainscow (2021) en este mismo monográfico se puede encontrar el planteamiento general del modelo, así como los principales resultados, implicaciones, impacto y conclusiones a las que se ha llegado con su aplicación en las escuelas de diferentes países europeos. En este trabajo se mostrará cómo se ha llevado a cabo en las escuelas españolas, así como las lecciones aprendidas del proceso. 
En concreto, los objetivos que se han planteado han sido: a) mejorar las prácticas de los docentes implicados con la finalidad de asegurar el aprendizaje y la participación de todo su alumnado y b) analizar y valorar el proceso seguido para su utilización sistemática en las escuelas como estrategia de mejora docente continua.

\section{Método}

Se ha llevado a cabo un proceso de investigación-acción colaborativa con dos ciclos durante un curso escolar. Cada ciclo de investigación-acción ha constado de tres fases: planificar las lecciones, enseñanza y evaluación. En todas han participado los docentes y los estudiantes investigadores.

\section{Participantes}

Se ha contado con la participación de 6 CEIP (Centros de Educación Infantil y Primaria) de la Comunidad de Madrid. En cada centro han colaborado profesorado y alumnado. Respecto al profesorado se ha contado con la participación de 18 docentes $(77,8 \%$ mujeres y 22,2\% hombres), contándose con profesorado de los seis cursos de primaria, así como de las asignaturas de Lengua y Literatura, Matemáticas, Inglés, Ciencias Naturales, Ciencias Sociales, Música y Educación Física. Un total de 201 estudiantes de Educación Primaria se implicaron en el estudio, de los cuales 117 pertenecían a las aulas en las se implantó la estructura colaborativa mencionada. Los 84 alumnos restantes pertenecían a aulas del mismo curso y de los mismos centros, y sirvieron de grupo control para analizar la eficacia de la propuesta. Además, un total de 52 estudiantes ejercieron el rol de investigadores (entre 8 y 9 por escuela).

La media de edad de estos alumnos fue de 9.05 años, con un rango de 6 a 13 años, pertenecientes a distintos niveles educativos. El 52.6\% de los participantes eran hombres y el $47.4 \%$ eran mujeres. Asimismo, tres investigadores de la universidad han participado como amigos críticos.

\section{Instrumentos}

Para recoger la información del desarrollo y análisis de las "lecciones de investigación" se diseñaron e implementaron diferentes estrategias:

- Observaciones de aula por parte de los estudiantes investigadores. El foco general de estas observaciones fue si sus compañeros están atentos, si hacen lo que el profesor pide, si están interesados por la clase o molestan a otros, si participan haciendo preguntas o respondiendo, si colaboran, si están aburridos y si hay compañeros que están solos o tristes.

- Entrevistas de los estudiantes investigadores a algunos alumnos después de cada lección para consultar sobre la comprensión de los contenidos de la lección, su interés, participación, aprendizaje y qué mejorarían. Los guiones de estas entrevistas fueron acordados por los estudiantes investigadores.

- Grupos de discusión entre estudiantes, docentes y profesorado de la universidad en dos momentos, para planificar la lección y después de su impartición. La reflexión de estos grupos giró en torno a la valoración de la lección en términos de aprendizaje y participación de todos los estudiantes, qué se puede mejorar y qué características debería tener una clase para ser inclusiva. La estructura de estos grupos de discusión fue propuesta por el profesorado de la universidad. 
- Cuestionario para medir la implicación del alumnado (Messiou et al., 2021). Este cuestionario consta de 15 ítems en escala Likert, y se centra en analizar la implicación de los alumnos en las actividades de aprendizaje, sus sentimientos de autonomía y pertenencia al grupo y su percepción de oportunidades para participar. El cuestionario utilizado en este estudio ha modificado la escala Likert a 5 puntos (de 1-nada a 5-muchísimo). Este cambio no altera la estructura interna de la escala. Este mismo cuestionario fue aplicado a alumnado de otros grupos no participantes en las lecciones de investigación, pertenecientes a los mismos cursos y centros educativos, que sirvieron de grupos control.

Además de los instrumentos anteriores, en cada escuela los estudiantes decidieron cómo recoger información previa de cada grupo respecto a qué les ayuda a aprender y participar en clase. Estos instrumentos variaron de una escuela a otra (entrevistas, cuestionarios, dibujos, votaciones, etc.) ${ }^{1}$.

\section{Procedimiento}

Cada ciclo del proceso de investigación-acción ha constado de tres fases: planificar, enseñar y evaluar. En todas han participado los docentes y los estudiantes investigadores. Previamente, en cada centro se formó un trío de profesores que voluntariamente aceptaron participar en el proyecto. Estos docentes: a) han colaborado en el diseño de las "lecciones de investigación”, b) han llevado a cabo observaciones mutuas durante la impartición de estas lecciones, y c) han discutido, junto con los estudiantes investigadores, los resultados de las observaciones, identificando aspectos a mejorar a través de los grupos de discusión llevados a cabo al final de las lecciones de investigación.

El profesorado involucró a un grupo de estudiantes investigadores. El criterio de selección fue su representatividad en cuanto a la diversidad existente en el aula, prestando una atención especial a aquellos que se encontraban en una situación de mayor vulnerabilidad. El profesorado informó a estos estudiantes del proyecto, y ellos finalmente decidieron si querían participar en el mismo. Este grupo de estudiantes recibió formación a través de talleres para ser investigadores ${ }^{2}$. En estos talleres decidieron la manera en la que recogerían información sobre las opiniones de sus compañeros y compañeras de clase.

Los estudiantes investigadores de cada escuela; a) recogieron evidencias sobre qué ayudaba a sus compañeros y compañeras a aprender y participar más en las clases, y sobre su percepción respecto al impacto de las clases en estos dos aspectos, aprendizaje y participación y, b) participaron en las discusiones relacionadas con el diseño de las lecciones de investigación.

Los investigadores de la universidad han tenido un papel de observador, facilitador y han contribuido al análisis de las evidencias (datos obtenidos de entrevistas, observaciones, cuestionarios) de todo el proceso de investigación. Los procesos de diálogo entre docentes y alumnos (grupos de discusión) se grabaron en video y/o audio para su posterior

\footnotetext{
1 Ver una muestra de instrumentos en (Students" Voices Toolkit): https://reachingthehardtoreach.eu/pupil-voice-tool-kit/

${ }^{2}$ La información detallada del proceso de trabajo con docentes y estudiantes se puede consultar en https://reachingthehardtoreach.eu/publication/
} 
transcripción y análisis, contando en todo momento con los correspondientes consentimientos informados de los participantes.

El análisis de la información recogida se llevó a cabo para cada centro educativo, partiendo de la transcripción de las grabaciones, la lectura preliminar de las mismas, elaboración de las categorías de análisis, codificación, asignación de cada fragmento a las categorías temáticas y de contenido.

Antes de empezar la investigación-acción y al final de la misma, se pidió que cumplimentasen el cuestionario de implicación del alumnado los grupos participantes en las lecciones de investigación y los grupos controles de los diferentes centros educativos y cursos. Esto se llevó a cabo en el aula y se aclararon a los alumnos todas las dudas. Se consideró pertinente aplicar el cuestionario también a un grupo control igualado en curso y de los mismos centros educativos, para poder valorar también de manera cuantitativa la eficacia de la propuesta.

\section{Resultados}

Dada la naturaleza tan amplia del proyecto, los resultados se presentan en torno a la percepción de los docentes y los estudiantes respecto a: a) las mejoras introducidas por los docentes para que sus lecciones "lleguen a todos y todas", b) el empoderamiento de los estudiantes investigadores, c) la colaboración docente como palanca para la mejora, y c) la percepción de cambio didáctico por parte del alumnado.

Estos resultados se han derivado de las herramientas recogidas en el apartado de instrumentos, cuyos informantes han sido docentes, estudiantes investigadores y los compañeros y compañeras de las clases en las que se llevaron a cabo las lecciones de investigación.

\subsection{Mejoras introducidas por los docentes para que sus lecciones "Ileguen a todos y todas"}

El profesorado ha reconocido que el proceso llevado a cabo ha contribuido a mejorar sus clases, sobre todo, desde el punto de vista de prestar una mayor atención a aquellos alumnos o alumnas que reconocen que les cuesta más que aprendan y participen. Se observa que sus "prácticas mejoradas" han tenido como referencia las voces de sus estudiantes y las evidencias recogidas (un análisis pormenorizado de estas mejoras se encuentra en Sandoval y otros 2020). Así, por ejemplo, han introducido actividades que a veces pueden implicar movimiento (pues hay estudiantes que lo necesitan): canciones, juegos interactivos sobre los contenidos que se están enseñando, entre otras. También han diversificado sus explicaciones con esquemas, mapas mentales, etc., incluyendo, asimismo, diferentes formas de evaluar.

Además, se han introducido actividades encaminadas a que todos los estudiantes tengan oportunidades para participar en clase, lo que supone, por ejemplo, cuidar los niveles de dificultad o el apoyo prestado en determinadas actividades. También se ha hecho hincapié en la importancia de que todos los alumnos sean conscientes de que el profesor les presta atención, que son importantes para ellos: moviéndose por la clase y revisando o preguntando cómo lo están haciendo y no preguntando solo a los que levantan la mano como ocurre con frecuencia. Los docentes reconocieron la importancia de dar tiempo a las y los estudiantes que no son tan rápidos o necesitan pensar más sus respuestas. 
Tengo la suerte de ser la tercera pues después de haberos visto ha sido importante- $\mathrm{Ya}$ estoy cambiando cosas. Cuando lo ves desde atrás, ves los que están perdidos y ahora estoy más pendiente. (Profesor)

Yo también ahora me estoy moviendo más por la clase. (Profesor)

Por otro lado, se han hecho mejoras relacionadas con la estructuración de la clase, introduciendo ciertas rutinas o anticipando el desarrollo previsto: comienzo, desarrollo propiamente dicho (con un equilibrio de actividades teniendo en cuenta la diversidad de estudiantes del aula) y cierre.

Se ha producido un incremento importante de las actividades que implican cooperación entre el alumnado, siendo esta petición una de las más repetidas tras las diversas consultas. Sus efectos fueron comentados por los estudiantes,

... hay que aprender a ser compañeros (...) aprender a colaborar, es tan importante la función del ayudante como el que recibe ayuda (...) pero todos deben tener la oportunidad de ayudar. (Estudiante investigador)

y por los docentes,

Hemos comprobado como a veces ayuda mucho, en lugar de explicar algo nosotros los profesores, que lo hagáis vosotros Muchas veces viene bien tirar de vosotros para que expliquéis, no para explicar solo al compañero de al lado, sino a la clase. (Profesor)

\subsection{Empoderamiento de los estudiantes investigadores}

Los docentes han cambiado su percepción del papel del alumnado, viéndole ahora como potenciales aliados para la mejora de sus prácticas.

Porque es verdad que algunas personas necesitan una cosa y otros otras (...) pero también hay cosas comunes a todos, como que es importantísimo lo que habéis dicho, trabajar en equipo, también individualmente. Pero necesitamos escucharos y da igual la edad que tengáis. Porque un niño de 6 o 7 años tiene formas de decir cómo aprende mejor (...) es muy interesante lo que estáis diciendo y necesitamos escucharlo para mejorar como profesores y profesoras. (Profesor)

Los estudiantes también reconocen aprendizajes valiosos y efectos positivos sobre su propia autoestima, parte de lo cual tiene que ver con el hecho, precisamente, de apreciar que sus profesores han considerado sus aportaciones:

He aprendido a trabajar en equipo, pues hemos tenido que trabajar en equipo todos juntos y he aprendido que cada niño piensa diferente. (Estudiante investigador)

Por otro lado, gracias a esta experiencia son también más sensibles a los ritmos de aprendizaje y a las necesidades de sus compañeros, han aprendido a comprenderles mejor y respetarles más:

Poder demostrar a todos los niños que todos somos igual de inteligentes. (Estudiante investigador)

Ayudar a otras personas que lo necesitan pues igual hay un niño que no se siente tan cómodo en la clase porque tal vez no le gusta la manera de enseñar y tú puedes ayudarle a que siga la clase. (Estudiante investigador)

Asimismo, cabe destacar el impacto en la valoración que los estudiantes investigadores hacen de su propia escuela, al ver el interés de su profesorado por mejorar su docencia.

A mí me ha gustado hablar con los niños, interactuar con los niños y ver cómo las profesoras han querido mejorar para que todos los niños aprendan mejor. (Estudiante investigador) 
También su actitud como estudiantes ha cambiado. La observación del comportamiento de sus compañeros y cómo este afectaba al desarrollo de la clase, les ha ayudado a tomar conciencia del papel que tienen en las clases y de su propia actitud hacia el aprendizaje.

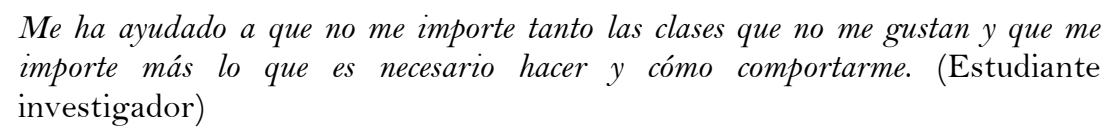

\subsection{La colaboración docente como palanca para la mejora}

Se ha valorado especialmente que el proyecto haya promovido y facilitado la colaboración entre docentes, a través de la posibilidad de observarse y aprender unos de otros.

$$
\begin{aligned}
& \text { He disfrutado, he aprendido mucho observando y reflexionado con mis compañeras, } \\
& \text { qué importante es tener esa posibilidad. (Profesor) }
\end{aligned}
$$

Este "pararse a pensar" no puede hacerse en vacío, sino que son necesarias evidencias, a modo de catalizadores para la mejora (proporcionadas en este caso tanto por los estudiantes como por sus propios compañeros). También se ha hecho evidente que los cambios son el efecto de la convergencia de una serie de actitudes personales de los docentes y de condiciones escolares, que también se han puesto de manifiesto, en ocasiones por su ausencia:

\section{(el cambio) No es magia y no surge de la nada. Ocurre creando las condiciones propicias. (Profesor)}

En este sentido hay que resaltar que el equipo de docentes debe estar seriamente comprometido con el "cuidado" de todos sus estudiantes y con la actitud de que "nadie se quede atrás".

Los profesores nos debemos adaptar a todos nuestros alumnos, pues educamos personas por lo que todos difieren entre ellos y debemos lograr su máximo desarrollo en todas las facetas. (Profesor)

Esta actitud profesional choca, la inmensa mayoría de las veces, con las rutinas del día a día:

$$
\begin{aligned}
& \text {.. y ver cómo podemos equilibrarlo como nuestro día a día, a veces, aunque queramos } \\
& \text { no llegamos a todos, pero esto debe ser una prioridad. (Profesor) }
\end{aligned}
$$

\subsection{Percepción de cambio didáctico por parte del alumnado}

Para medir la eficacia del programa, se analizaron las respuestas al cuestionario cumplimentado al inicio y al final de las lecciones de investigación, tanto por el grupo participante como por el grupo control. Para la realización de los análisis, se aplicó estadística no paramétrica.

En primer lugar, se realizó análisis de Wilcoxon tanto para el grupo control como para el grupo participante en las lecciones de investigación, a fin de ver su evolución entre la primera medida antes del inicio de estas lecciones (pre) y la segunda medida una vez terminadas (post).

En el grupo en el que se llevaron a cabo las lecciones de investigación los resultados mostraron diferencias estadísticamente significativas entre las puntuaciones pre y post $(Z$ $=-4.290, \mathrm{p}=0,000$; Pre: Media $=4.04, \mathrm{Sd}=0,42$; Post: Media $=4,20, \mathrm{Sd}=0,45)$. Las puntuaciones totales de participación fueron superiores después del desarrollo de estas lecciones que, en la medida tomada previamente, lo que significa que mejoró la percepción por parte de este alumnado. 
En el grupo control no se encontraron diferencias significativas entre los dos momentos en los que se hizo la recogida de datos, en lo que respecta al cuestionario total, por lo que no varió significativamente su percepción durante ese tiempo.

En segundo lugar, para profundizar en estos resultados, el análisis se enfocó solo en el grupo participante (test de Wilcoxon) para ver en qué ítems se apreciaba evolución antes y después de las lecciones de investigación. En el Cuadro 1 se muestran las medias y desviaciones típicas de cada ítem en los dos momentos pre y post del grupo participante en las lecciones de investigación.

\section{Cuadro 1}

Medias y desviaciones típicas de cada ítem (grupo participante en las lecciones de investigación)

\begin{tabular}{lcc}
\hline & Pre & Post \\
\hline He participado en las actividades de clase & $4,13(0,878)$ & $4,44(0,759)$ \\
Lo que dije en la clase hoy fue valorado & $419(0,988)$ & $4,21(0,973)$ \\
Me sentí incómodo/a por estar en clase & $2,80(1,724)$ & $2,30(1,635)$ \\
Estaba interesado en aprender & $4,70(0,590)$ & $4,69(0,684)$ \\
Trabajé tanto como pude & $4,20(0,838)$ & $4,38(0,738)$ \\
Lo que hice fue valorado & $4,26(0,922)$ & $4,48(0,853)$ \\
Después de terminar mi trabajo comprobé si era correcto & $3,94(1,235)$ & $4,01(1,141)$ \\
Me costó entender todo lo que el profesor/a me explicó & $2,90(1,355)$ & $2,78(1,558)$ \\
Mis compañeros me escucharon & $3,87(1,098)$ & $4,39(0,815)$ \\
Tuve algo que decir sobre lo que estábamos haciendo & $3,56(1,444)$ & $3,44(1,568)$ \\
Las cosas que aprendí tenían sentido para mí & $4,41(0,827)$ & $4,55(0,769)$ \\
Pude intercambiar opiniones con mis compañeros/as & $3,88(1,271)$ & $3,98(1,267)$ \\
Mi profesor/a me escuchó & $4,72(0,717)$ & $4,84(0,443)$ \\
Fingí que estaba trabajando & $2,18(1,664)$ & $1,71(1,332)$ \\
Me sentí seguro entre mis compañeros & $4,45(0,990)$ & $4,53(0,833)$ \\
\hline
\end{tabular}

Los resultados mostraron diferencias estadísticamente significativas entre la medida pre y post-intervención en los ítems "He participado en las actividades de clase" ( $Z=-3.829$, $\mathrm{p}=0,000)$, "Mis compañeros me escucharon" $(Z=-4.428, p=0,000)$ y "Trabajé tanto como pude" $(Z=-2.117, p=0,034)$. En todos ellos, las puntuaciones se incrementan tras la actuación llevada a cabo, indicando una mayor percepción de participación, de escucha y de trabajo. También se encontraron diferencias significativas, pero en sentido contrario, en los ítems "Me sentí incómodo por estar en clase" $(Z=-2.839, p=0,005)$ y "Fingí que estaba trabajando" $(Z=-3.123, p=0,002)$. En ambos, las puntuaciones disminuyen significativamente indicando que, después del desarrollo del programa, el alumnado se siente menos incómodo en clase y finge menos que trabaja.

\section{Conclusiones}

Ainscow (2005) ha hablado de la importancia de conocer y elegir bien las palancas que tienen mayor capacidad para promover en las escuelas cambios sostenibles con un horizonte inclusivo. El modelo de las "lecciones de investigación" utilizado en este proyecto resulta, sin lugar a duda, una de esas potentes palancas en la línea de lo apuntado en estudios previos (Messiou et al, 2016). Que lo sean no significa que necesariamente, aplicadas a las rutinas establecidas, las transformen, de la noche a la mañana, en formas de enseñar y evaluar que permitan el aprendizaje y la participación de todo el alumnado. 
Como han mostrado los resultados obtenidos, la creación de estructuras colaborativas en las escuelas resulta un recurso didáctico y formativo útil en los centros educativos para aumentar el aprendizaje y la participación de todo el alumnado. Lo que surge de todas las experiencias de los centros fue el reconocimiento de que los propios estudiantes han jugado un rol decisivo en la medida que, entre otras cosas, han participado en diálogos con el profesorado, apoyando sus opiniones o propuestas en evidencias recogidas gracias a la utilización de diferentes herramientas como observación y entrevistas. Pruebas que, además, fueron acordadas por los propios estudiantes y analizadas junto con los docentes implicados. De hecho, la colaboración de los estudiantes y el profesorado en materia de planificación, enseñanza y evaluación tuvo efectos positivos en la mejora docente respecto a cómo enseñar, así como en la comprensión de los contenidos y en la calidad de las experiencias de aprendizaje por parte de su alumnado. Además, el profesorado percibía a los estudiantes como colaboradores y, en parte, responsables del desarrollo de las sesiones, porque en mayor o menor medida, estos habían contribuido de forma activa al diseño de esas lecciones.

Pero para que esto funcione, el proceso que se ha mostrado no puede ser algo improvisado. Se requiere de una planificación realista y sostenible. Debe tener vocación de convertirse en un "proyecto de centro" que vaya permeando en su cultura, lo que requiere del ineludible apoyo del equipo directivo acompañado de un liderazgo distribuido (Harris et al, 2018). Además, hay que difundir en el centro lo logrado y ofrecer una retroalimentación frecuente del proceso, porque de lo contrario podría instalarse la percepción de que no está pasando nada o nada está cambiando. Esa retroalimentación compartida también debe servir para ir valorando todo lo positivo que, de modo colateral, pueda estar generando esta dinámica.

Por otro lado, hay que tener una "genuina actitud de querer escuchar" lo que el alumnado puede decirnos sobre cómo hacer que las clases sean más inclusivas, lo que implica tomarse en serio lo que puedan decir y, como consecuencia de ello, estar dispuestos a cambiar. Si los estudiantes no perciben esta actitud, desconfiarán del profesorado y dejarán de participar (Hill et al., 2004).

No obstante, lo más difícil es no privilegiar en este entramado las voces de los que se perciben más capaces de expresar su sentir o parecer, en detrimento de aquel alumnado al que, por distintas razones, le puede costar más hacerse oír y escuchar (Mocker y Groundwater-Smith, 2015). Esto requerirá dedicar tiempo, entre otras cosas, a enseñarles las habilidades sociales y comunicativas necesarias para compartir sus ideas y emociones, así como a revisar los procedimientos utilizados y la participación de todos los alumnos y alumnas en los mismos.

El desarrollo del proyecto nos ha ido mostrando cambios concretos, pero consistentes, tanto en las concepciones del profesorado respecto a la diversidad de su alumnado como, en sus prácticas, en las actitudes y valoración positiva de sus estudiantes para acompañar al docente en esta y otras tareas. Pero también en el alumnado ha impactado positivamente en lo relacionado con su percepción de participación, en su interés e implicación en las clases. Ello ha sido así al menos, durante el tiempo en el que el profesorado ha tenido disponible el "andamiaje" del resto de participantes, así como el estímulo y la motivación que supone la participación en este tipo de proyectos. Creemos firmemente que este es un buen camino para seguir, aunque también es un sendero largo y tortuoso que, por esas mismas razones, la clave de su sostenibilidad y generalización estará en la capacidad de 
las administraciones educativas competentes y en los equipos directivos de los colegios, para crear y cuidar las "condiciones escolares" que han permitido facilitar los esperanzadores resultados que se han mostrado en este trabajo.

\section{Agradecimientos}

Este trabajo se enmarca en el proyecto financiado por la Unión Europea titulado "Reaching the 'the hard to reach' (ReHaRe): respuestas inclusivas a la diversidad a través del diálogo alumnos-profesores (programa Erasmus+ K2), coordinado por la Dra. Kiki Messiou de la Universidad de Southampton.

\section{Referencias}

Ainscow, M. (2005). Developing inclusive education systems: What are the levers for change? Journal of Educational Change, 6(2), 109-124. https://doi.org/10.1007/s 10833-005-1298-4

Ainscow, M., Dyson, A., Goldrick, S. y West, M. (2013). Promoviendo la equidad en educación. Revista de Investigación en Educación, 11 (3), 44-56.

Agencia Europea para las Necesidades Especiales y la Inclusión Educativa. (2012). Perfil profesional del docente en la educación inclusiva. Agencia Europea para el Desarrollo de la Educación del Alumnado con Necesidades Educativas Especiales.

Fielding, M. (2018). Radical democracy and student voice in secondary schools. En J. Feu y O. Prieto-Flores (Eds.), Democracy and education in the $21^{\text {st }}$ century: The articulation of new democratic discourses and practices (pp. 69-85). Peter Lang

Harris, J., Carrington, S. y Ainscow, M. (2018). Promoting equity in schools. Collaboration, inquiry and ethical leadership. Routledge. https://doi.org/10.4324/9781315105659

Hill, M., Davis, J., Prout, A. y Tisdall, K. (2004). Moving the participation agenda forward. Children and Society, 18(2), 77-96. https://doi.org/10.1002/chi.819

Martín, E. y Cervi, J. (2006). Modelos de formación docente para el cambio de concepciones en los profesores. En J. I. Pozo, N. Scheuer, M. P. Pérez Echevarría, M. Mateos, E. Martín y M. De la Cruz (Eds.), Nuevas formas de pensar la enseñanza y el aprendizaje. Concepciones de profesores y alumnos (pp. 419-434). Graó.

Messiou, K. (2019). Understanding marginalisation through dialogue: a strategy for promoting the inclusion of all students in schools. Educational Review, $71(3)$, 306-317. https://doi.org/10.1080/00131911.2017.1410103

Messiou, K. y Ainscow, M. (2020). Inclusive inquiry: Student-teacher dialogue as a means of promoting inclusion in schools. British Educational Research Journal, 46(3), 670-687. https://doi.org/10.1002/berj.3602

Messiou, K. y Ainscow, M. (2021). Inclusive inquiry: An innovative approach for promoting inclusion in schools. Revista Latinoamericana de Educación Inclusiva, 15(2), art 4.

Messiou, K., Ainscow, M., Echeita, G., Goldrick, S., Hope, M., Paes, I., Sandoval, M., Simon, C. y Vitorino, T. (2016). Learning from differences: A strategy for teacher development in respect to student diversity. School Effectiveness and School Improvement, 27(1), 45-61. https://doi.org/10.1080/09243453.2014.966726

Messiou, K., Hedegaard-Sørensen, L., Ulvseth, H. Gasteiger-Klicpera, B., Bešić E. et al., (2021). Documento guía para el seguimiento de la implicación del alumnado en la Investigación Inclusiva. University of Southampton. 
McLeskey, J., Waldron, N.L., Spooner, F. y Algozzine, B. (2014). Handbook of Effective Inclusive Schools. Research and practice. Routledge. https://doi.org/10.4324/9780203102930

Mockler, N. y Groundwater-Smith, S. (2015). Engaging with student voice in research, education and community: Beyond legitimation and guardianship. Springer. https://doi.org/10.1007/978-3-319-01985-7

Morris, D. (2019). Student voice and teacher professional development. Knowledge exchange and transformational learning. Palgrave McMillan. https://doi.org/10.1007/978-3-030-23467-6

Norwich, B., Benham-Clarke, S. y Goei, S. L. (2021). Review of research literature about the use of lesson study and lesson study-related practices relevant to the field of special needs and inclusive education. European Journal of Special Needs Education, 36(3), 309-328. https://doi.org/10.1080/08856257.2020.1755929

Porter, G. y Towell, D. (2019). Advancing school transformation from within. Inclusive education canada/centre for inclusive futures. UNESCO.

Sandoval, M., Simón, C. y Echeita, G. (2020). ¿Qué me ayuda a aprender y participar? Herramientas para recoger las voces de los estudiantes. Revista de Educación Inclusiva, 13(1), 12-27.

Simón, C., Echeita, G. y Sandoval, M. (2018). Incorporating students' voices in the "Lesson Study" as a teacher-training and improvement strategy for inclusion. Culture and Education, 3O(1), 215-225. https://doi.org/10.1080/11356405.2017.1416741

Susinos, T., Ceballos, N. y Saiz, A. (2018). Cuando todos cuentan. Experiencias de participación de estudiantes en las escuelas. La Muralla.

Soto, E., Serván, M. J., Peña, N. y Pérez A. I. (2019). Action research through lesson study for the reconstruction of teachers" practical knowledge. A review of research at Malaga University (Spain), Educational Action Research, 27(4), 527-542. https://doi.org/10.1080/09650792.2019.1610020

Tomlinson, C. (2015). Teaching for excellence in academically diverse classrooms. Society, 52(3), 203-209. https://doi.org/10.1007/s12115-015-9888-0

UNESCO. (2015). Education 2030: Incheon declaration and framework for action for the implementation of sustainable development goal 4. UNESCO.

UNESCO. (2020a). Towards inclusion in education: Status, trends and challenges. UNESCO.

UNESCO. (2020b). Global education monitoring report, 2020: Inclusion and education. UNESCO.

\section{Breve CV de los/as autores/as}

\section{Cecilia Simón}

Profesora Titular del Departamento Interfacultativo de Psicología Evolutiva y de la Educación de la Universidad Autónoma de Madrid (UAM). Es co- coordinadora del grupo de investigación "Equidad, diversidad y educación inclusiva" (EQUIDEI) de la UAM. Forma parte de Catalizadores para la Educación Inclusiva (Inclusion International) y ha colaborado con entidades como la OEI y la Agencia Europea para las Necesidades Educativas Especiales y la Inclusión Educativa. Sus intereses de investigación se relacionan con el desarrollo de una educación más inclusiva, así como con la relación escuela, familia y comunidad. Ha participado en múltiples proyectos de ámbito nacional e internacional sobre estos temas, siendo IP de alguno de ellos. Entre los más recientes se encuentran "Responding to diversity by engaging with students' voices: a strategy for 
teacher Development"; "Teaching diverse learners in school subjects"; "Reaching the hard to reach: Inclusive responses to diversity through child-teacher dialogue"; "European network of inclusive universities" así como "Análisis y valoración del proceso de educación inclusiva del alumnado con Trastornos del Espectro del Autismo desde educación infantil hasta la universidad: la participación social como eje de análisis" (EITEA). Email: cecilia.simon@uam.es

ORCID ID: https://orcid.org/0000-0002-1795-9109

\section{Gerardo Echeita}

Profesor Titular del Departamento de Psicología Evolutiva y de la Educación de la Facultad de Psicología de la Universidad Autónoma de Madrid (UAM). Ha trabajado en el Ministerio de Educación de España (1986/1996) y ha sido contratado como experto o consultor, en el campo de la educación inclusiva para diferentes proyectos promovidos por organizaciones internacionales como UNESCO, OCDE, OEI y la Agencia Europea para las Necesidades Educativas Especiales y la Educación Inclusiva. Tiene más de cien publicaciones, entre artículos, capítulos de libros o libros y ha participado en numerosas actividades formativas, así como en conferencias, congresos y jornadas, en particular en el ámbito latinoamericano. Email: gerardo.echeita@uam.es

ORCID ID: https://orcid.org/0000-000 1-8682-5342

\section{Marta Sandoval}

Maestra, orientadora y profesora Contratada Doctora de la Facultad de Formación del Profesorado y Educación de la Universidad Autónoma de Madrid. Coordinadora del Grupo de investigación sobre Educación Inclusiva y diversidad y miembro de la Cátedra UNESCO en Educación para la justicia social. Su interés investigador se centra en la formación del profesorado en inclusión, la colaboración docente y las voces de los estudiantes en la mejora escolar. Ha participado hasta el momento en ocho proyectos competitivos internacionales. Email: marta.sandoval@uam.es

ORCID ID: https://orcid.org/0000-0003-1931-1872

\section{$M^{\mathrm{a}}$ José de Dios}

Doctora en Psicología, Profesora del Departamento de Psicología Evolutiva y de la Educación de la Facultad de Psicología de la Universidad Autónoma de Madrid Ha desarrollado su carrera docente en diversas universidades españolas, como la Universidad Internacional de La Rioja, la Universidad Pontificia de Comillas y la Universidad Camilo José Cela, habiendo desarrollado gran parte de su carrera en ésta última. Su carrera investigadora ha estado centrada en el estudio de la infancia y adolescencia en el contexto escolar, enfocada en tres líneas de trabajo complementarias: En primer lugar, comenzó su labor investigadora estudiando el desarrollo de procesos básicos como la motivación de logro en el ámbito educativo desde una perspectiva sociocultural, tema en el que centró su tesis doctoral. En segundo lugar, fue realizando nuevas investigaciones sobre la influencia de distintos contextos de enseñanza en el desarrollo de estrategias motivacionales y de aprendizaje en los alumnos, siendo investigadora principal de varios proyectos en esta línea. En tercer lugar, ha participado desde el inicio de su carrera en investigaciones sobre el proceso de desarrollo de alumnos con distintas alteraciones y la influencia del ambiente escolar en su inclusión educativa. En total, ha participado en más 
C. Simón et al.

de veinticinco proyectos competitivos y no competitivos que han dado lugar a más de un centenar de publicaciones y presentaciones a congresos sobre estos temas. Email: mjose.dedios@uam.es

ORCID ID: https://orcid.org/0000-0003-4701-3135 